\title{
DEUTSCHE DICHTUNG IN EPOCHEN
}

\section{Ein literaturgeschichtliches Lesebuch}

\author{
Herausgegeben \\ von Walter Kißling \\ bearbeitet \\ von Achim Block, Helmut Hoffacker, \\ Peter Kohrs, Paul Schmitt \\ und Ulrich Vossen
}

J.B. Metzlersche Verlagsbuchhandlung

Stuttgart 


\section{Deutsche Dichtung in Epochen}

Ein literaturgeschichtliches Lesebuch für den Deutschunterricht auf der Oberstufe herausgegeben von

Dr. Walter Kißling, Walldorf

bearbeitet von

Dr. Achim Block, Präsident des Landesprüfungsamts Niedersachsen, Göttingen

Helmut Hoffacker, Oberstudienrat, Bremen

Peter Kohrs, Studiendirektor, Paderborn-Elsen

Paul Schmitt, Studienrat, Dötlingen-Busch

Ulrich Vossen, Oberstudiendirektor, Kaarst

Die einzelnen Kapitel wurden verfaßt:

Mittelalter, Barock, Weimarer Republik von Achim Block

Aufklärung, Biedermeier und Vormärz, Realismus, Faschismus und Exil von Helmut Hoffacker

Naturalismus, Bundesrepublik, DDR von Peter Kohrs

Empfindsamkeit und Sturm und Drang, Klassik, Zwischen Klassik und Romantik,

Romantik, Expressionismus von Paul Schmitt

Humanismus und Reformation, Barock, Symbolismus und Impressionismus von Ulrich Vossen

Einbandgestaltung: Willy Löffelhardt

Gestaltung: Axel Eberhard

Redaktion: Dr. Walter Kißling, Walldorf

Gabriele Ernst, Jorinde Assemann

ISBN 978-3-476-20470-7

ISBN 978-3-476-98792-1 (eBook)

DOI 10.1007/978-3-476-98792-1

Dieses Werk einschließlich aller seiner Teile ist urheberrechtlich geschützt.

Jede Verwertung außerhalb der engen Grenzen

des Urheberrechtsgesetzes ist ohne Zustimmung des Verlages

unzulässig und strafbar.

Das gilt insbesondere für Vervielfältigungen,

Übersetzungen, Mikroverfilmungen und die Einspeicherung

und Verarbeitung in elektronischen Systemen.

(C) 1989 Springer-Verlag GmbH Deutschland

Ursprünglich erschienen bei J. B. Metzlersche Verlagsbuchhandlung und Carl Ernst Poeschel Verlag GmbH in Stuttgart 1989 


\title{
INHALTSVERZEICHNIS
}

\author{
"Deutsche Dichtung in Epochen«: Zur Didaktik, Konzeption und Verwendung. \\ Statt eines Vorworts \\ Seite Is
}

\section{Deutsche Literatur des Mittelalters}

Seite 17

Unbekannte Verfasser

Die Merseburger Zaubersprüche I8

Das Hildebrandslied I9

Das Wessobrunner Gebet 2 I

Otfried von Weissenburg

Evangelienbuch $\mathbf{2 2}$

Unbekannter Verfasser

Aus dem Abrogans 28

Noker (von Zwifalten?)

Memento mori $3 \mathrm{I}$

Pfaffe Konrad

Das Rolandslied 34

Wolfram von Eschenbach

Parzival 35

Unbekannte Verfasser

Das Nibelungenlied 43

Dû bist mîn 6I

Der von Kürenberc

>Ich zôch mir einen valken` 6 I

Der Burcgrave von Rietenburc

Ich hôrte wîlent sagen ein mære 6I

Her Dietmar von Eist

Slâfst du, friedel ziere? 62

Her Reinmar

*Nur die eine 62

*Geraubter Kuß 63

*Botschaft an den Geliebten 64

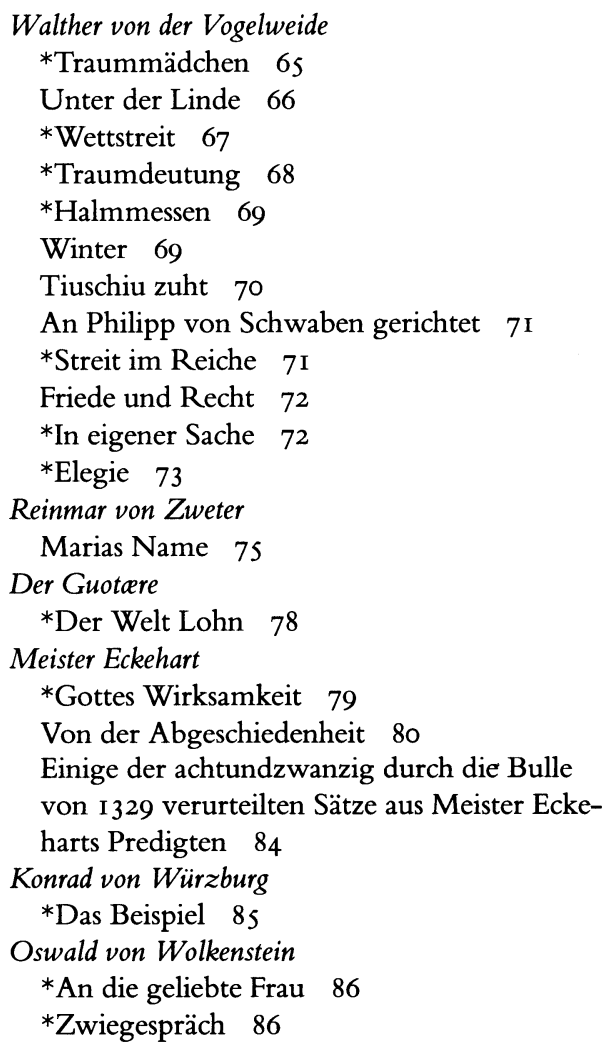

Die mit * markierten Titel bezeichnen von den Lesebuch-Autoren gewählte Textüberschriften im Unterschied zu den von den Dichtern selbst formulierten Überschriften.

Bei mittelalterlicher Lyrik werden Titel mit* gekennzeichnet, wenn sie von Editoren oder Lesebuch-Autoren gewählt sind. Überschriften ohne* nehmen dort Formulierungen aus dem jeweiligen Text auf. 


\section{Anbruch der Neuzeit: Humanismus und Reformation}

Seite 88

Anklage gegen den Tod

Johannes von Tepl

Der ackermann 89

Narren und Weise

Sebastian Brant

Das Narren schyff 95

Die Bibel: "verteutscht"

Übersetzungen von Markus I4,3-IO IOO

Martin Luther

Ein sendbrieff D.M. Lutthers. Von Dol-

metzschenn IO2

Um Wahrheit und Freiheit

Ulrich von Hutten

*Vorrede I07

Ain new Lied herr Vlrichs von Hutten 108

Thomas Murner

Von dem grossen Lutherischen narren 109

Unbekannter Verfasser

Karsthans II I

\section{Der Kampf der Bauern}

Conz Annahans und andere

Das püntisch Liedlein II 3

Thomas Müntzer

Hochverursachte Schutzrede und Antwort wider das geistlose, sanftlebende Fleisch zu Wittenberg II 4

An die Allstedter IIs

An die Mühlhäuser II6

Martin Luther

Wider die Mordischen und Reubischen Rotten der Bawren 1 I 8
Sebastian Münster

Der vierdte Stand $\quad 120$

Johannes Agricola

Teütsche Sprichwörter I2I

Kirchenlieder, Volkslieder, Meistersang

Martin Luther

Aus tieffer not 122

$\mathrm{Nu}$ bitten wyr den heyligen geyst $\quad 122$

Ein feste burg ist vnser Gott $\quad$ I23

Hans Sachs

Sant Petter mit der gais $\quad 124$

Unbekannte Verfasser

Entlaubet ist der walde I25

*Verschneiter Weg I25

Isbruck ich muß dich lassen I26

*Mühlrad I26

*Die Königskinder 126

Wo sol ich mich hin keren $\quad$ I28

Sant Marten wollen loben wir 129

Lehrreiches und Kurzweiliges

Martin Luther

Etlich Fabeln aus dem Äsopo

verdeudscht I3O

Jörg Wickram

Das Rollwagenbüchlin I 3 I

Hans Sachs

Ein spiel mit funf personen:

Der dot im stock $\quad$ I33

\section{Volksbücher}

Unbekannte Verfasser

Ein kurtzweilig lesen von

Dyl Vlenspiegel I39

Das Lalebuch I4I

Historia vnd Geschicht

Doctor Johannis Faustj I43 


\section{Barock}

Seite I 49

\section{Die Welt: ein leerer Wahn?}

\section{Andreas Gryphius \\ Vanitas! Vanitatum Vanitas! I so \\ Es ist alles eitell I 52 \\ Christian Hoffmann von Hoffmannswaldau \\ Die Welt I 52 \\ Andreas Gryphius \\ An eine hohen Standes Jungfraw I 53 \\ Anton Ulrich von Braunschweig \\ Sterb-Lied I 53 \\ Daniel Casper von Lohenstein \\ Umbschrifft eines Sarches I 54 \\ Paul Fleming \\ Herrn Pauli Flemingi der \\ Med.Doct. Grabschrifft I 54}

Sei dennoch unverzagt!

Paul Fleming

An Sich I5s

Andreas Gryphius

Abend I55

Paul Gerhardt

Táglicher Abendgesang 156

Hans Jacob Christoffel von Grimmelshausen

Komm Trost der Nacht I 57

Catharina Regina von Greiffenberg

Auf Gottes seltsame Geist-Regirung $\quad$ I 58

Auf eben selbige 158

Martin Rinckart

Tisch-Gebetlein I 59

Angelus Silesius

Cherubinischer Wandersmann I 59

Johann Caspar Schade

Gott 160

Johann Christian Günther

Trostaria I6I

Martin Opitz

Liedt I6I

Johann Rist

Auff die nunmehr angekommene kalte

Winterszeit $\quad$ I62

Liebe und galantes Spiel

Martin Opitz

Sonnet 162
Georg Greflinger

Sein gutes Gefångnư $B \quad$ I63

Filip von Zesen

An seine lieb - und holdsálige

Adelmund 163

Paul Fleming

*An Elsgen $\quad 164$

Simon Dach

Trewe Lieb' ist jederzeit $\mathrm{Zu}$

gehorsamen bereit I65

Mey-Liedchen 166

Martin Opitz

Ein Anders (Lied) $\quad$ I67

Georg Greflinger

Eine Jungfraw vber ihres Liebsten

Abreyse ${ }^{16} 67$

Johann Christian Günther

Abschiedsaria 168

Johann Rist

Daphnis bekưmmerte Liebes-Gedancken/Als er

bey seiner Galatheen nicht seyn konte I69

Johann-Hermann Schein

O Sternen Äugelein I7I

Paul Fleming

Wie Er wolle gekússet seyn $\quad 172$

Christian Hoffman von Hoffmannswaldau

*Albanie 173

Georg Greflinger

*Gegensätze $\quad 174$

Volkstümliches

Unbekannte Verfasser

Mütterlein, was soll ich thun? I75

Es geht eine dunkle Wolk

herein 176

Ein schónes Mayenlied $\quad 176$

Ich bin ein freyer Bauers-Knecht! I78

Ist es nit ai Elendt lieba $\quad$ I79

Die Welt als Schauplatz

Hans Jacob Christoffel von Grimmelshausen Lebensbeschreibung Der Ertzbetrigerin und Landstorrtzerin Courasche I8 I

Andreas Gryphius

Absurda Comica. Oder Herrn Peter

Squentz/Schimpff-Spiel 193 
Höfisches Leben

Johann Rist

Das AllerEdelste Leben der gantzen

Welt 20I

Daniel Casper von Lohenstein

Agrippina 206

Filip von Zesen

Assenat 219
Deutsche Sprache und Dichtkunst

Martin Opitz

Buch von der Deutschen

Poeterey 225

Johann Michael Moscherosch

Gesichte Philanders von

Sittewald 23I

\section{Aufklärung}

Seite 234

Selbstbestimmung der Epoche

Christoph Martin Wieland

Sechs Antworten auf sechs Fragen 235

Funktion der Literatur

Johann Christoph Gottsched

Versuch einer kritischen Dichtkunst vor die

Deutschen 238

Johann Jakob Breitinger

Von dem Wunderbaren und dem Wahr-

scheinlichen 240

Lyrik: Nützlichkeit des Natürlichen

Barthold Hinrich Brockes

Das Blümlein Vergißmeinnicht 242

Albrecht von Haller

Natur und Gott 244

Roman: Geschichte und Gegenwart

Christoph Martin Wieland

Geschichte der Abderiten 246
Drama: Vernünftiges Handeln

Johann Elias Schlegel

Die stumme Schönheit 25I

Gotthold Ephraim Lessing

Nathan der Weise 255

Fabula docet

Gotthold Ephraim Lessing

Die Geschichte des alten Wolfs in siebe:

Fabeln 26I

Christian Fürchtegott Gellert

Die Nachtigall und die Lerche 263

Aphorismen zur Sozialkritik

Georg Christoph Lichtenberg

Aphorismen 265

\section{Empfindsamkeit und Sturm und Drang}

Seite 267

Johann Wolfgang Goethe

Prometheus 269

Friedrich Gottlieb Klopstock

Der Zürchersee 270

Johann Wolfgang Goethe

Im Herbst 272

Es schlug mein Herz 273

Friedrich Schiller

Laura am Klavier 274
Gottfried August Bürger

Mollys Wert 275

Der Bauer 275

Christian Friedrich Daniel Schubart

Deutscher Freiheitsgeist 276

Der Gefangene 277

Friedrich Gottlieb Klopstock

Die États Généraux 278

Mein Irrtum 279 
Matthias Claudius

Kriegslied 280

Abendlied 28I

Johann Heinrich Jung-Stilling

Heinrich Stillings Jugend.

Eine wahrhafte Geschichte 282

Johann Wolfgang Goethe

Die Leiden des jungen Werthers 284
Gottfried August Bürger

Verhör einer Kindsmörderin $\quad 287$

Karl Philipp Moritz

Anton Reiser. Ein psychologischer

Roman 289

Friedrich Maximilian Klinger

Sturm und Drang 29I

Friedrich Schiller

Über Bürgers Gedichte 295

\section{Klassik}

Seite 297
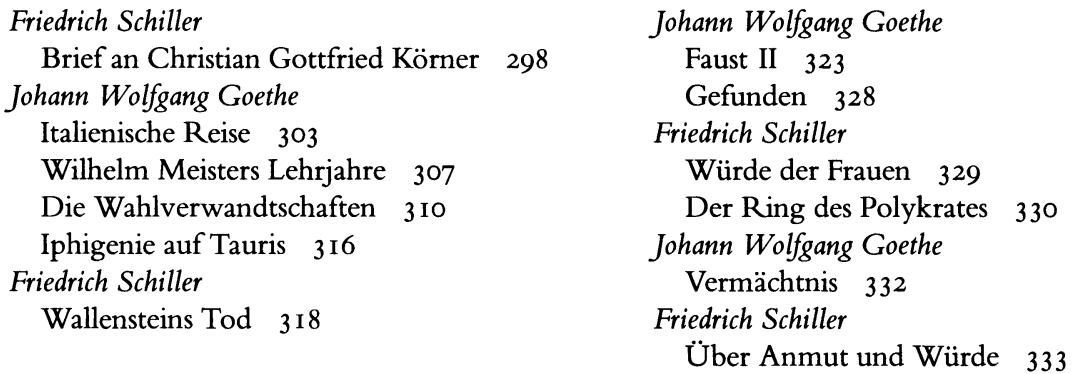

\section{Zwischen Klassik und Romantik: Hölderlin, Jean Paul, Kleist}

Seite 336

\author{
Friedrich Hölderlin \\ Abendphantasie 337 \\ Mnemosyne 338 \\ Ernst Zimmer \\ Brief an Hölderlins Mutter 340 \\ Friedrich Hölderlin \\ Wenn aus dem Himmel ... 342
}

An Zimmern 343

Jean Paul

Doktor Katzenbergers Badereise 343

Heinrich von Kleist

Anekdoten. Ausgewählt aus den "Berliner

Abendblätter(n)« 348

Prinz Friedrich von Homburg 350

\section{Romantik}

Seite 355

Novalis

Wenn nicht mehr Zahlen und Figuren 356 Jacob und Wilhelm Grimm

Kinder- und Hausmärchen 356

Adelbert von Chamisso

Die Weiber von Winsperg 357

Achim von Arnim / Clemens Brentano

Aus: Des Knaben Wunderhorn 358
Friedrich Baron de la Motte Fouqué Undine. Eine Erzählung 360 Ernst Theodor Amadeus Hoffmann Undine. Zauberoper in 3 Akten 363 Klein Zaches genannt Zinnober 365 Lebensansichten des Katers Murr nebst fragmentarischer Biographie des Kapellmeisters Johannes Kreisler in zufälligen Makulaturblättern 368 
Novalis

Heinrich von Ofterdingen 373

Bettina von Arnim

Über die Günderode 376

Joseph von Eichendorff

Ahnung und Gegenwart 379

Friedrich Schlegel

Briefe auf einer Reise durch die Niederlande,

Rheingegenden, die Schweiz und einen Teil

von Frankreich 382

Clemens Brentano

Lorelay 384
Joseph von Eichendorff

Sehnsucht 386

Der Abend 386

Mondnacht 386

Memento mori! 387

Theodor Körner

Lützow's wilde Jagd $\quad 387$

Justinus Kerner

Der Wanderer in der Sägemühle 388

An Sie im Alter 390

Joseph von Eichendorff

Erlebtes 390

\section{Biedermeier und Vormärz \\ Seite 39I}

Biedermeierliche Lebenswelt

Annette von Droste-Hülshoff

Mondesaufgang 39I

Nikolaus Lenau

Das Mondlicht 393

Eduard Mörike

Er ist's 393

Gesang Weylas 393

Auf eine Lampe 394

September-Morgen 394

Denk es, o Seele 394

Karl Leberecht Immermann

Die Epigonen 395

Jeremias Gotthelf

Der Bauernspiegel 397

Franz Grillparzer

Ein treuer Diener seines Herrn 400

Ferdinand Raimund

Der Verschwender 406

Poesie und Politik

Ludolf Wienbarg

Ästhetische Feldzüge. Worte der

Zueignung 410

Der Beschluß des Bundestages vom

Io. Dezember I 835 zum Verbot der Schriften des Jungen Deutschland 4I I

\section{Politisierung der Lyrik}

Heinrich Hoffmann von Fallersleben

Lied der Deutschen 4 II2

Heinrich Heine

Die Tendenz 4I2
Nachtgedanken 4I3

Die schlesischen Weber $4 \mathrm{I} 3$

Georg Herwegh

Wiegenlied 4I4

Kein Preußen und kein Österreich 4IS

Ferdinand Freiligrath

Abschiedswort der Neuen Rheinischen

Zeitung 4IS

Revolutionsgeschichte auf der Bühne

Georg Büchner

Dantons Tod 4I7

Christian Dietrich Grabbe

Napoleon oder Die hundert Tage 425

Prosa des bürgerlichen Erwerbslebens und des Abenteuers

Georg Weerth

Humoristische Skizzen aus dem deutschen

Handelsleben 430

Heinrich Heine

Aus den Memoiren des Herren von

Schnabelewopski 435

Demokratische Publizistik:

Die Waffe der Kritik

Friedrich Engels

Das industrielle Proletariat 438

Wilhelm Wolff

Das Elend und der Aufruhr in Schlesien 440 


\section{Realismus}

\section{Seite 444}

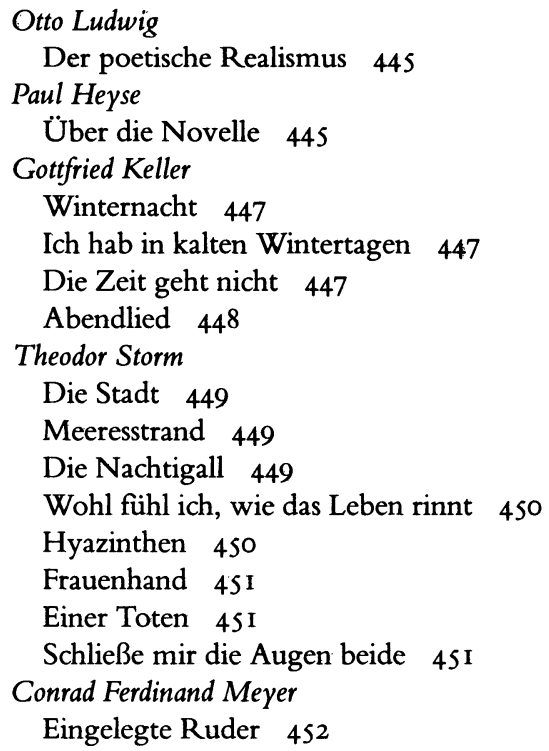

Im Spätboot $\quad 452$

Zwei Segel 452

Der römische Brunnen 452

Friedrich Hebbel

Agnes Bernauer 453

Ludwig Anzengruber

Der Meineidbauer 456

Wilhelm Raabe

Chronik der Sperlingsgasse 459

Adalbert Stifter

Der Nachsommer 462

Gottfried Keller

Der grüne Heinrich 465

Theodor Fontane

Effi Briest 469

Der Stechlin 474

Friedrich Nietzsche

Apollinisch und Dionysisch 477

Karl Hillebrand

Über Sprachvermengung 479

\section{Naturalismus - Beginn der Moderne \\ Seite 482}

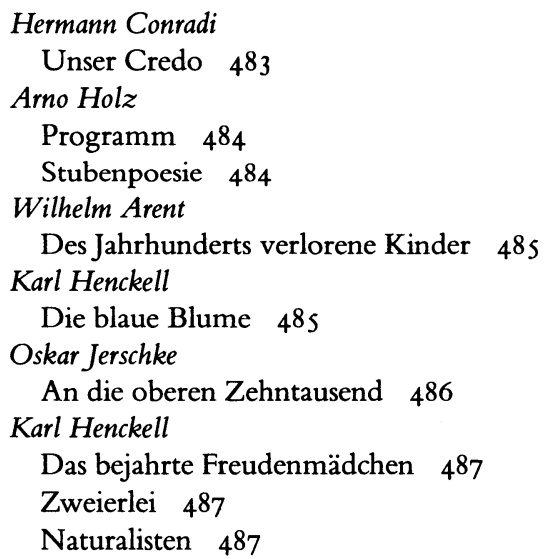

Arno Holz

Phantasus 488

Aus dem Gedichtzyklus "Phantasus» 488

Revolution der Lyrik 490

Arno Holz/Johannes Schlaf

Die Familie Selicke 49I

Gerhart Hauptmann

Hanneles Himmelfahrt 494

Ludwig Thoma

Magdalena 498

Detlev von Liliencron

Der Narr 500

Den Naturalisten 503

\section{Symbolismus und Impressionismus \\ Seite 504}

Wort und Welt

Stefan George

Das Wort sos
Des Sehers Wort 506

Hugo von Hofmannsthal

Was ist die Welt? 506

Ein Brief 507 


\section{Erlebnis und Ahnung}

Stefan George

Wir schreiten auf und $a b$ im reichen

flitter 513

Gustav Falke

Zwei $5 \mathrm{I} 3$

Hugo von Hofmannsthal

Die beiden $5 \mathrm{I} 4$

Christian Morgenstern

Morgenfahrt 514

Krähen bei Sonnenaufgang 5 IS

Rainer Maria Rilke

Die Flamingos 515

Der Panther 516

Das Karussell $\$ 17$

Herbsttag 5 I 8
Friedrich Nietzsche

Abschied 5 I 8

*Venedig $5 \mathrm{I} 8$

Innenreich und äußeres Leben

Hugo von Hofmannsthal

Ballade des äußeren Lebens 5 I 9

Manche freilich... 520

Rainer Maria Rilke

Denn sieh: sie werden leben... 520

*Die großen Städte 522

Stefan George

Die tote Stadt 523

Detlev von Liliencron

Die Musik kommt 524

Arthur Schnitzler

Leutnant Gustl 524

\section{Expressionismus}

Seite $53 \mathrm{I}$

Alfred Lichtenstein

Die Dämmerung 532

Georg Heym

Das Grundbuchamt 532

Jakob van Hoddis

Weltende 533

Johannes Robert Becher

Ödipus-Zyklus II. An den Vater 534

Mädchen-Zyklus I. Franziska 535

Gerrit Engelke

Tagebuchblätter aus dem Kriege 536

Georg Trakl

Grodek 536

August Stramm

Wache 537

Sturmangriff 537

Patrouille 537

Ernst Jünger

In Stahlgewittern $\quad 538$

Ernst Stadler

Heimkehr 538
Else Lasker-Schüler

Sulamith 540

Ein alter Tibetteppich $\quad 542$

Karl Kraus

Anmerkung zu »Ein alter Tibetteppich" 542

Wilhelm Klemm

Der Bettler 542

Albert Ehrenstein

Zigeuner 543

Gottfried Benn

Morgue 545

Georg Trakl

Gesang des Abgeschiedenen 546

Rudolf Leonhard

Der tote Liebknecht 547

René Schickele

Bei der Einfahrt in den Hafen von

Bombay 547

Carl Einstein

Bebuquin oder die Dilettanten des

Wunders 548 


\section{Literatur in der Zeit der Weimarer Republik}

Seite 550

Kurt Tucholsky

Dämmerung 550

Karl Kraus

Die letzten Tage der Menschheit 554

Franz Kafka

Brief an den Vater 556

Vor dem Gesetz $56 \mathrm{I}$

Rainer Maria Rilke

Aus den Sonetten an Orpheus 562

Bertolt Brecht

Ballade von den Abenteurern 562

Erinnerung an die Marie A. 563

Alles Neue ist besser als alles Alte 564
Die Nachtlager 565

Ich, der ich nichts mehr liebe 565

Über die Bauart langdauernder Werke 566

Marieluise Fleißer

Pioniere in Ingolstadt 566

Briefe aus dem gewöhnlichen Leben 567

Oskar Maria Graf

Des Pudels Kern 569

Alfred Döblin

Berlin Alexanderplatz 57I

Robert Musil

Der Mann ohne Eigenschaften 575

Heinrich Mann

Unfall einer Republik 58I

\section{Literatur in der Zeit des Faschismus und des Exils}

Seite 585

Positionen

Klaus Mann

Klaus Mann an Gottfried Benn 586

\section{Gottfried Benn}

Antwort an die literarischen Emigranten $\quad 588$

Lyrik

Baldur von Schirach

Dem Führer 592

Will Vesper

Dem Führer 592

Erwin Guido Kolbenheyer

Dank 593

Josef Weinheber

Treue 593
Bertolt Brecht

Gedanken über die Dauer des Exils 594

An die Nachgeborenen 595

Rückkehr 597

\section{Drama}

Hanns Johst

Schlageter 598

Bertolt Brecht

Mutter Courage und ihre Kinder 60I

Prosa

Josefa Berens-Totenohl

Der Femhof 605

Anna Seghers

Das siebte Kreuz 608

\section{Nachkriegszeit und Bundesrepublik}

Seite 613

Nach dem Kriege

Günter Eich

Inventur 6I5

Paul Celan

Todesfuge 6I5
Werner Bergengruen

Die letzte Epiphanie 617

Wolfgang Borchert

Draußen vor der Tür 617

Dann gibt es nur eins! 621 
Heinrich Böll

Bekenntnis zur Trümmerliteratur 623

Beunruhigungen und Warnungen

Max Frisch

Der andorranische Jude 626

Günter Eich

Träume 628

Paul Celan

Espenbaum 634

Ingeborg Bachmann

Die gestundete Zeit 635

Ernst Jandl erstarrt gegenüber 635

Hans Magnus Enzensberger Ins Lesebuch für die Oberstufe 636

Marie-Luise Kaschnitz

Beschwörung 636

Wende in der deutschen Nachkriegsprosa

Günter Grass

Die Blechtrommel 637

Uwe Johnson Mutmaßungen über Jakob 64I

Heinrich Böll

Ansichten eines Clowns 643

Siegfried Lenz

Deutschstunde 646

\section{Vielfalt lyrischer Aussageweisen}

Gottfried Benn

Rosen 650

Nur zwei Dinge 650

Probleme der Lyrik 65I

Nelly Sachs

Das ist der Flüchtlinge Planetenstunde 652

Hilde Domin

Im Regen geschrieben 652

Ingeborg Bachmann

Freies Geleit 653

Paul Celan

Fadensonnen 653

Günter Bruno Fuchs

Nach der Haussuchung 654

Hans Magnus Enzensberger

Konjunktur 654
Günter Grass

Kinderlied 655

Franz Josef Degenhardt

Macht euch nichts vor 655

Erich Fried

Beim Wiederlesen eines Gedichtes von Paul

Celan 657

Wolf Wondratschek

Hotel "Zum Deutschunterricht" 658

Michael Krüger

Literatur 659

Guntram Vesper

Der letzte Winter 659

\section{Literatur als Dokumentation}

Peter Weiss

Die Ermittlung - Oratorium in I I Gesängen 660

Günter Wallraff

Am Fließband 667

Sinnsuche in einer Welt voller Widersprüche: Poesie der Groteske und des

Absurden

Friedrich Dürrenmatt

Weihnacht 669

Christoph Meckel

Weltende 670

Friedrich Dürrenmatt

Die Physiker 67I

Wolfdietrich Schnurre

Der Schattenfotograf 675

Beziehungen

Arno Schmidt

Die Vorsichtigen 675

Gabriele Wohmann

Der Antrag 677

Peter Bichsel

San Salvador 68I

Peter Handke

Besitzverhältnisse 682

Franz Xaver Kroetz

Oberösterreich 685 


\section{Nachkriegszeit und DDR}

Seite 687

\section{Ausgangssituation}

\section{Peter Huchel}

Deutschland 689

Stephan Hermlin

Die Zeit der Wunder 690

Bruno Apitz

Nackt unter Wölfen 69I

Aufbau, Abgrenzung, Agitation

Bertolt Brecht

Aufbaulied 696

Johannes Robert Becher

Nationalhymne der Deutschen

Demokratischen Republik 697

Kuba

J. W. Stalin 698

Louis Fürnberg

Lied von der Partei 699

Werner Bräunig

Greif zur Feder, Kumpel! 700

Jürgen Köditz

Dreherin 702

Heinz Kahlau

Alle Sätze 702

Jan Koplowitz

Song von der Verfassung 702

Brecht und seine Nachfolge

Bertolt Brecht

Deutschland $1952 \quad 703$

Für Helene Weigel 703

Kleines Organon fur das Theater 704

Buckower Elegien 706

Heiner Müller

Der Lohndrücker 707

Volker Braun

Die Kipper 7I I

Lyrik als Möglichkeit individueller

Aussagen

Günter Kunert

Individueller Ausbruchsversuch 715

So soll es sein 715
Johannes Bobrowski

Pruzzische Elegie 716

Sprache 717

Peter Huchel

Winterpsalm 7 I7

Reiner Kunze

Sensible Wege 718

Karl Mickel

Maischnee 718

Sarah Kirsch

Die Luft riecht schon nach Schnee 7 I9

Kritische Tendenzen auf dem Boden des

Sozialismus

Peter Huchel

Der Garten des Theophrast 719

Reiner Kunze

Das Ende der Kunst 720

Franz Fühmann

Die Weisheit der Märchen 721

Günter Kunert

Tagträume in Berlin und andernorts 722

Kurt Bartsch

Sozialistischer Biedermeier 722

Helga M. Novak

Abgefertigt 723

Kurt Bartsch

Größe 724

Der Redner 724

Mut 724

Reiner Kunze

Appell 724

Christa Wolf

Der geteilte Himmel 725

"Öffnungen" - Beginn der Ära Honecker

Volker Braun

Kontinuität 73I

Stefan Heym

5 Tage im Juni 73 I

Volker Braun

Mitteilung an die reifere Jugend 738

Konfrontation, Verweigerung, Protest

Reiner Kunze

Beweggründe 738 
Wolf Biermann

Ermutigung 739

Der Brief der DDR-Künstler nach der Ausbürgerung Wolf Biermanns 740

Jürgen Fuchs

Die Lüge 740

Gerald K. Zschorsch

Expression 74I

Bernd Jentzsch

Das Verlangen 74I

Jurek Becker

Die Strafe 74I

Frauen schreiben über Frauen

Sarah Kirsch

Zwillinge 743
Angela Stachowa

Ich bin ein Kumpel 745

Die junge Schriftstellergeneration:

Lebensgefühle, Wünsche, Hoffnungen

Volker Braun

Unvollendete Geschichte 747

Matthias Biskupek

Fragebogen 750

Dieter Kerschek

Schein Kontrolle 750

Gudula Ziemer

Juni. Jaguar. Einzelgänger 751

Martin Stephan

Der Vagabund 75I

\section{Literaturhinweise}

Seite 753

Sekundärliteratur

Seite 753

Primärliteratur

Seite $76 \mathrm{I}$

Autoren- und Quellenverzeichnis

Seite 766

Personen- und Werkregister

Seite 788 